\title{
Why some tumours trigger neovascularisation and others don't: the story thus far
}

\author{
Omanma Adighibe', Russell D. Leek', Marta Fernandez-Mercado ${ }^{2,3}$, Jiangting Hu' ${ }^{1}$, Cameron Snell',
} Kevin C. Gatter ${ }^{1}$, Adrian L. Harris ${ }^{4}$ and Francesco Pezzella ${ }^{1 *} \mathbb{D}$

\begin{abstract}
Background: Angiogenesis is not essential for tumours to develop and expand, as cancer can also grow in a nonangiogenic fashion, but why this type of growth occurs is unknown. Surprisingly, our data from mRNA transcription profiling did not show any differences in the classical angiogenic pathways, but differences were observed in mitochondrial metabolic pathways, suggesting a key role for metabolic reprogramming. We then validated these results with mRNA profiling by investigating differential protein expression via immunohistochemistry in angiogenic and non-angiogenic non-small cell lung cancers (NSCLCs).
\end{abstract}

Methods: Immunohistochemical staining for 35 angiogenesis- and hypoxia-related biomarkers were performed on a collection of 194 angiogenic and 73 non-angiogenic NSCLCs arranged on tissue microarrays. Sequencing of P53 was performed with frozen tissue samples of NSCLC.

Results: The non-angiogenic tumours were distinguished from the angiogenic ones by having higher levels of proteins associated with ephrin pathways, mitochondria, cell biogenesis, and hypoxia-inducible factor 1 (HIF1) regulation by oxygen and transcription of HIF-controlled genes but lower levels of proteins involved in the stroma, cell-cell signaling and adhesion, integrins, and Delta-Notch and epidermal growth factor (EGF)-related signaling. However, proteins classically associated with angiogenesis were present in both types of tumours at very comparable levels. Cytoplasmic expression of P53 was strongly associated with non-angiogenic tumours. A pilot investigation showed that P53 mutations were observed in $32.0 \%$ of angiogenic cases but in $71.4 \%$ of non-angiogenic tumours.

Conclusions: Our observations thus far indicate that both angiogenic and non-angiogenic tumours experience hypoxia/HIF and vascular endothelial growth factor (VEGF) pathway protein expression in a comparable fashion. However, angiogenesis does not ensue in the non-angiogenic tumours. Surprisingly, metabolic reprogramming seems to distinguish these two types of neoplastic growth. On the basis of these results, we raise the hypothesis that in some, but not in all cases, initial tissue remodeling and/or inflammation could be one of the secondary steps necessary to trigger angiogenesis. In the non-angiogenic tumours, in which neovascularisation fails to occur, HIF pathway activation could be the driving force toward metabolic reprogramming.

Keywords: Cancer, Angiogenesis, Hypoxia, Blood vessels

\footnotetext{
*Correspondence: francesco.pezzella@ndcls.ox.ac.uk

1 Radcliffe Department of Medicine, Nuffield Division of Laboratory

Science, John Radcliffe Hospital, University of Oxford, Oxford OX3 9DU, UK

Full list of author information is available at the end of the article
} 


\section{Background}

In 1927, Otto Warburg described what would be called the "Warburg effect," in which tumour cells exhibited characteristic changes in metabolism, particularly the use of glycolysis rather than oxidative phosphorylation, despite the presence of adequate amounts of oxygen [1, 2]. Warburg believed that this process was the actual cause of neoplastic transformation [3].

Tumour development is now known to be driven by genetic damage. However, mutations in some metabolic enzymes, such as succinate dehydrogenase (SDH) and fumaratehydratase $(\mathrm{FH})$, both parts of the tricarboxylic acid (TCA) cycle, can drive neoplastic transformation, and intermediate products of metabolism can also promote neoplastic progression [4].

Proliferating cancer cells have a high energy requirement to maintain homeostatic cellular processes. The shift in energy production to aerobic glycolysis, while allowing for more rapid production of adenosine triphosphate (ATP), yields far less energy than oxidative phosphorylation: there are two net molecules of ATP per glucose molecule in glycolysis versus 36 molecules of ATP via oxidative phosphorylation [5].

The reasons for the glycolytic energy dependence of proliferating tumour cells are still being debated. Initially, it was believed that the mitochondria in tumours were intrinsically defective. However, it was determined that tumour mitochondria are actually functional, retaining the capacity for oxidative phosphorylation and consuming oxygen at similar rates to normal tissues [6], although it should be appreciated that a degree of variability in mitochondrial activities exists across different neoplasms. Alternatively, high rates of glycolysis might be co-selected with factors that promote the expression of hypoxia-related genes (such as those required for angiogenesis) as an oxygen-independent energy source. Finally, increased intermediate products of glycolysis can easily be shunted into the biosynthetic pathways required for serine and nucleotide synthesis [7].

According to Folkman's original theory [8], the onset of hypoxia in tumour triggers angiogenesis, which in turn is essential for supplying neoplastic cells with nutrients and oxygen and evacuating metabolic waste and carbon dioxide. The best understood hypoxia signaling mechanism is the stabilization and post-transcription activation of the hypoxia-inducible factor (HIF) proteins, which lead to the activation of many different pathways, including the vascular endothelial growth factor (VEGF) pathway. The VEGF pathway prompts and supports neoangiogenesis and glycolysis. Hypoxia-inducible pathway activation also has other effects, which include reducing the activity of mammalian target of rapamycin (mTOR), which in turn can reignite autophagy and promote survival under stress [9].
HIF is a heterodimer of an alpha subunit that is unstable in normoxia and a constitutively present and stable beta subunit. The hypoxia activation of HIF causes the heterodimer to bind to DNA at specific locations, called hypoxic response elements (HREs), eliciting the transcriptional up-regulation of genes required to respond appropriately to hypoxia [9].

In addition to triggering the VEGF pathway, the ubiquitously expressed HIF1 isoform promotes the transcription of glucose transporter 1 (GLUT1), which activates glucose transport inside the cell, lactate dehydrogenaseA (LDH-A), which is involved in the glycolytic pathway, erythropoietin (EPO), which enhances erythropoiesis, and nitric oxide synthase (NOS), which promotes angiogenesis and vasodilatation [9].

HIF1 also prevents the entry of pyruvate into the TCA cycle by inducing the expression of pyruvate dehydrogenase kinase 1 (PDK1), thus altering the expressed isoform of cytochrome $c$ and inhibiting mitochondrial biogenesis. This process causes reduced levels of oxygen consumption and a shift away from oxidative phosphorylation. Interestingly, HIF1 can also be activated under normoxic conditions by a variety of oncogenic pathways, such as phosphatidylinositol-4,5-bisphosphate 3-kinase catalytic subunit alpha (PIK3CA), and by mutations in von Hippel-Lindau tumour suppressor (VHL), SDH, and FH [10].

In the classic angiogenic pathway, VEGF binds to VEGF receptor 2 (VEGFR2) on endothelial cells, increasing the expression of the Notch ligand Deltalike 4 (DLL4) on the same cells. DLL4 then binds to its receptor Notch on the adjacent endothelium. Further expression of VEGFR2 and VEGFR1, as well as a smaller amount of VEGFR3, then follows, leading to triggering/amplification of the downstream phospholipase $\mathrm{C}$ familyy (PLCY)-protein kinase C (PKC)-Raf kinaseMAP kinase-ERK kinase (MEK)-mitogen-activated protein kinase (MAPK) pathway, concomitantly prompting cell proliferation and cell survival throughout the phosphoinositide 3-kinase (PI3 K)/protein kinase B (AKT) pathway [11].

The switch to glycolysis in neoplasia was, according to Warburg, irreversible [3], yet a more complex picture has emerged over the last decade. There have been observed instances in which oxidative phosphorylation predominates during neoplastic transformation [12]. This variation between OxPhos and glycolysisin cancer cells has been increasingly linked to specific disturbances in cell signaling pathways [13].

Additionally, tumours of the same genetic lineage can develop different metabolic adaptations depending on the host tissue from which they arise, suggesting that the stromal environment might play a crucial role in shaping the 
metabolic profile [14]. The different molecular mechanisms being postulated to explain this variability of the Warburg effect include the following: inhibition of pyruvate dehydrogenase (PDH) by PDK1, reduction of mitochondrial biogenesis and inhibition of oxidative phosphorylation, both are caused by P53 inactivation and mutations [15].

Warburg raised two important issues: first, how tumour cells are supplied with glucose; and second, how they are supplied with oxygen [1]. Folkman's work addressed the latter question with the hypothesis that tumour growth is strictly angiogenesis-dependent [16]. The work undertaken to test this hypothesis led to the inclusion of "angiogenesis" as one of the hallmarks of cancer [8].

Although there is strong evidence that angiogenesis frequently occurs in cancer, we also now know that this event does not always occur. Indeed, some tumours, called "nonangiogenic tumours," can grow without triggering new vessel formation by co-opting preexisting vessels $[17,18]$.

Non-angiogenic growth was first identified by histology in primary and metastatic lung carcinomas because neoplastic cells filled the alveolar spaces, co-opting the pre-existing capillary network and exhibiting a characteristic "chicken-wire" appearance [17]. A gene expression signature for non-angiogenic non-small cell lung cancer (NSCLC) was published in 2005 [19]. Surprisingly, rather than the classic angiogenesis-related genes, the differentially expressed genes were involved in mitochondrial metabolism, transcription, protein synthesis, and the cell cycle. Lack of differential mRNA expression between tumour phenotypes was noted for genes classically associated with hypoxia and angiogenesis. This result suggested that the response to hypoxia does not necessarily trigger neovascularisation, as would be observed in angiogenic tumours, but could actually be dependent on the genetic background of neoplastic cells, and in some instances, it could lead to metabolic reprogramming [19]. We therefore postulated that the degree to which a tumour will rely on angiogenic or non-angiogenic growth could be associated with a variety of events, including hypoxia, pseudohypoxia, and metabolic re-programming.

In the first part of the present study, we investigated whether there were truly no differences in the expression of hypoxia-and angiogenesis-related proteins between angiogenic and non-angiogenic tumours, as suggested by mRNA profiling. We also investigated the degree of these proteins expression and the expression of some mitochondrial biogenesis proteins via immunohistochemistry. Notably high cytoplasmic P53 expression in non-angiogenic tumours, compared to angiogenic tumours, was found after completing the first part of this study. We therefore performed a second investigation, in which we examined and sequenced the $p 53$ gene in these tumours.

\section{Methods}

\section{Tissue specimens}

Clinical specimens of NSCLCs were obtained from a series of consecutive patients who underwent surgical treatment at the John Radcliffe Hospital, Oxford, UK. This collection had ethical committee approval (study number C02.216-The pathophysiology of human neoplasia). The tissues were formalin-fixed and paraffinembedded. Tissue microarrays were constructed using the Beecher Instrument MTA-1 manual arrayer (Beecher Instruments, Inc., Sun Prairie, WI, USA). Up to four suitable areas of appropriate tumour were chosen from a slide stained with hematoxylin and eosin (H\&E), avoiding areas of necrosis.

\section{Immunohistochemical staining of tissue sections}

The 4- $\mu \mathrm{m}$ sections were cut from paraffin blocks and mounted on glass histology slides. Non-specific protein binding was blocked by incubating with $2.5 \%$ normal horse serum (Vector Laboratories, Burlingame, CA, USA), and the primary antibody was then applied. Details of all of the antibodies used are presented in Table 1. Substitution of the primary antibody with phosphate-buffered saline (PBS) served as a negative control. The slides were then counterstained with hematoxylin for $20 \mathrm{~s}$.

\section{Scoring}

The immunohistochemical staining was scored for cytoplasmic and nuclear localization and, when present, for membrane staining. Two observers scored the slides. Intensity was scored on a scale of $0-3(0=$ no staining, $1=$ weak, $2=$ moderate, $3=$ strong staining). The percentage of positive cells was recorded on a scale from 0 to $4(1=1 \%-10 \%, 2=11 \%-50 \%, 3=51 \%-80 \%, 4=81 \%-$ $100 \%)$ or alternatively on a continuous scale from $0 \%$ to $100 \%$. The intensity and percentage values were then multiplied to provide a score called the "intensity percentage score" (IPS), the maximum score of which ranged from 12 to 300 [20].

Disputed scores were discussed and a consensus reached. For tissue microarrays, cores that did not contain tumour tissue or that were more than $50 \%$ incomplete were excluded. The number of cases scored for each marker was between a minimum of 73 and a maximum of 194 cases for angiogenic tumours and between a minimum of 48 and a maximum of 73 cases for non-angiogenic tumours.

\section{Statistical analysis}

To evaluate the association between protein biomarker expression and angiogenic status, the Mann-Whitney two-tailed non-parametric test was performed using 
Table 1 Antibodies used for immunohistochemistry

\begin{tabular}{|c|c|c|}
\hline Antigen & Clone & Source \\
\hline HIF1 & ESEE122 & Abcam (Cambridge, UK) \\
\hline HIF2 & EP109b & NDCLS (University of Oxford, John Radcliffe Hospital, Oxford, UK) \\
\hline CA9 & M75 & BioScience Slovakia s.r.o. (Bratislava, Slovakia) \\
\hline VEGFA & VG-1 & NDCLS \\
\hline TYMP & PGF44 & NDCLS \\
\hline KDR & FLK1(A3) & Santa Cruz Biotechnology, Inc. (Dallas, TX, USA) \\
\hline KDRp34 & $34 a$ & NDCLS \\
\hline $\mathrm{FlH}$ & $\mathrm{FlH162c/D6}$ & NDCLS \\
\hline PHD1 & $\mathrm{PHD} 112 / \mathrm{G}$ & NDCLS \\
\hline PHD2 & $366 \mathrm{G} / 76$ & NDCLS \\
\hline PHD3 & EG188c & NDCLS \\
\hline DLL4 & D4/37 & NDCLS \\
\hline TSP1 & 8A6B-TSP-1 & Leica Microsystems (UK) Ltd. and Novocastra Reagents (Milton Keynes, UK) \\
\hline CXCR4 & MAB 172 & R\&D System (Minneapolis, MN, USA) \\
\hline EPHB2 & AF496 & R\&D System \\
\hline EPHB3 & R\&D AF432 & R\&D System \\
\hline EPHB4 & R\&D AF446 & R\&D System \\
\hline SOD1 & $30 F 11$ & Novocastra \\
\hline $\mathrm{BCL2}$ & 124 & Dako (Cambridgeshire, UK) \\
\hline FOS & Polyclonal 27436 & Abcam \\
\hline EGF & EGF 10 & Abcam \\
\hline EGFR & F4 & Abcam \\
\hline BNIP3 & Ana40 & Sigma-Aldrich Company Ltd. (Dorset, UK) \\
\hline P53 & DO7 & Dako \\
\hline $\mathrm{Pl} 3$ & Ab 40755 & Abcam \\
\hline SP1 & SP1 polyclonal & Abcam \\
\hline STAT3 & E121-21 & Abcam \\
\hline LON & $20 \mathrm{H} 1$ & NDCLS \\
\hline MEF2D & MEF2D polyclonal & Abcam \\
\hline$J M Y$ & HMY117A & NDCLS \\
\hline TRAP1 & TR-1A & LabVision (TorsbySjöväg, Värmdö, Sweden) \\
\hline GST & GST3/GST pi & Abcam \\
\hline NCAM & IB6 & Novocastra \\
\hline $\mathrm{CHGA}$ & FLEX polyclonal & Dako \\
\hline SYP & 299 & Novocastra \\
\hline
\end{tabular}

HIF hypoxia-inducible factor; CA9 carbonic anhydrase 9; VEGFA vascular endothelial growth factor A; TYMP thymidine phosphorylase; KDR vascular endothelial growth factor receptor 2; KDRp34 vascular endothelial growth factor p34; FIH factor-inhibiting HIF; PHD prolyl hydroxylase dehydrogenase; DLL4 Delta-like 4; TSP1 thymidine phosphorylase 1; CXCR4 chemokine (C-X-C motif) receptor 4; EPH ephrin; SOD1 superoxide dismutase 1; BCL2 B cell lymphoma 2; FOS FBJ murine osteosarcoma viral oncogene homolog; EGF epidermal growth factor; EGFR epidermal growth factor receptor; BNIP3 BCL2/adenovirus E1B $19 \mathrm{kDa}$ interacting protein 3; PI3 peptidase inhibitor 3; SP1 Sp1 transcription factor; STAT3 signal transducer and activator of transcription 3; LON Lon protease; MEF2D myocyte enhancer factor 2D; JMY junctionmediating and regulatory protein, p53 cofactor; TRAP1 TNF receptor-associated protein 1; GST glutathione S-transferase; NCAM neural cell adhesion molecule; CHGA chromogranin A; SYP synaptophysin

GraphPad Prism statistical analysis software, version 4 (GraphPad Software Inc., San Diego, CA, USA).

\section{Pathway visualization}

Three lists were made: two of proteins more highly expressed in angiogenic or non-angiogenic tumours at least in one subcellular location and the third of proteins always equally expressed in all of their subcellular localizations (Table 2). To visualize the pathways associated with these proteins, each list was imported into the Webbased Enricher facility (http://amp.pharm.mssm.edu/ Enrichr/) [21]. The data were visualized from the online databases for gene ontologies (GO biological process and GO cellular component) and for pathways [Kyoto 
Table 2 Lists of proteins used for pathway visualization using the EnrichrWebd facility [21]

\begin{tabular}{|c|c|}
\hline Expression status & Proteins \\
\hline Equal expression in both tumour types & $\begin{array}{l}\text { HIF2, VEGFA, TYMP, KDR, KDRp34, FIH, PHD1, SOD1, EPHB4, BCL2, EGFR, FGF, } \\
\text { SP1, LON, MEF2D, RPSA a , CHGA, SYP }\end{array}$ \\
\hline $\begin{array}{l}\text { Higher expression in angiogenic tumours than in non-angiogenic tumours } \\
\text { in at least one subcellular location }\end{array}$ & $\begin{array}{l}\text { HIF1, PHD2, PHD3 (cytoplasmic), CXCRN, TSP, DLL4, BNIP3, PI3, EGF, FOS, } \\
\text { STAT3, ITGB3 }{ }^{\text {, ITGAV }} \text { a }\end{array}$ \\
\hline $\begin{array}{l}\text { Higher expression in non-angiogenic tumours than in angiogenic tumours } \\
\text { in at least one subcellular location }\end{array}$ & CA9, PHD3 (nuclear), EPHB2, EPHB3, NCAM, P53, TRAP1, JMY, GST \\
\hline
\end{tabular}

FGF fibroblast growth factor; RPSA ribosomal protein SA; ITGB3 integrin, beta 3; ITGAV integrin alpha-V; HIF hypoxia-inducible factor; CA9 carbonic anhydrase 9; VEGFA vascular endothelial growth factor $A ; T Y M P$ thymidine phosphorylase; $K D R$ vascular endothelial growth factor receptor $2 ; K D R p 34$ vascular endothelial growth factor p34; FIH factor-inhibiting HIF; PHD prolyl hydroxylase dehydrogenase; DLL4 Delta-like 4; TSP1 thymidine phosphorylase 1; CXCR4 chemokine (C-X-C motif) receptor 4; $E P H$ ephrin; SOD1 superoxide dismutase 1; BCL2 B cell lymphoma 2; FOS FBJ murine osteosarcoma viral oncogene homolog; EGF epidermal growth factor; EGFR epidermal growth factor receptor; BNIP3 BCL2/adenovirus E1B $19 \mathrm{kDa}$ interacting protein 3; PI3 peptidase inhibitor 3; SP1 Sp1 transcription factor; STAT3 signal transducer and activator of transcription 3; LON Lon protease; MEF2D myocyte enhancer factor 2D; JMY junction-mediating and regulatory protein, p53 cofactor; TRAP1 TNF receptor-associated protein 1; GST glutathione S-transferase; NCAM neural cell adhesion molecule; CHGA chromogranin A; SYP synaptophysin

a Data for RPSA are from Ref. [18] and for ITGB3 and ITGAV are from Ref. [23]

Encyclopedia of Genes and Genomes (KEGG) 2015, WikiPathways 2015, Reactome 2015, and Panther]. The results were visualized by bar graph sorted by combined score.

\section{P53 sequencing}

Genomic DNA was isolated from 33 specimens of NSCLC (25 angiogenic cases, seven non-angiogenic cases, and one undetermined case) and five specimens of peri-tumour lung tissues. The coding regions corresponding to exons 3-9 (amino acids 25-331) were sequenced. DNA was whole-genome amplified using GenomiPhi (GE Healthcare, Piscataway, NJ, USA). The primers for polymerase chain reaction (PCR) amplification and subsequent sequencing reactions are described in Table 3. PCR was performed using ThermoStart PCR Master Mix (Thermo Fisher Scientific, Waltham, MA, USA), following the manufacturer's protocol. PCR products were purified and bidirectionally sequenced using the BigDye Terminator cycle sequencing kit, version 1.1 (Applied Biosystems, Foster City, CA, USA), and an ABI 3100 Genetic Analyzer (Applied Biosystem, Paisley, UK). Sequence data were analyzed using Mutation Surveyor, version 3.25 (Softgenetics, State College, PA, USA). Predicted effects on protein were assessed in silico using PolyPhen2 software (http://genetics.bwh.harvard.edu/ pph2/). Fisher's two-sided exact test was performed to compare mutation frequencies in angiogenic versus nonangiogenic cases. All sequencing experiments were performed in duplicate.

\section{Results}

Immunohistochemical staining

The complete results for the cytoplasmic and membranous expression are reported in Table 4, and those for nuclear expression are reported in Table 5, while a summary of these results appears in Table 6 . The complete original results of ontology and pathway visualization appear in Additional file 1.

In Table 7, a selection of pathways shows that proteins expressed in both types of tumours are associated with angiogenesis-, VEGF-, and oxidative stress-related pathways. The non-angiogenic tumours are distinguished from the angiogenic tumours by having higher levels of proteins related to ephrin pathways, response to hypoxia, HIF1 regulation by oxygen, and transcription

Table 3 Primers for polymerase chain reaction (PCR) amplification and subsequent P53 sequencing reactions

\begin{tabular}{|c|c|c|c|}
\hline Primer & Sequence & Annealing temperature $\left({ }^{\circ} \mathrm{C}\right)$ & Amplicon length (bp) \\
\hline Exon $3-4$ & $\begin{array}{l}\text { Forward: GTGGGAAGCGAAAATTCCAT } \\
\text { Reverse: GCCAGGCATTGAAGTCTCAT }\end{array}$ & 60 & 506 \\
\hline Exon 5-6 & $\begin{array}{l}\text { Forward: TGTTCACTTGTGCCCTGACT } \\
\text { Reverse: TTAACCCCTCCTCCCAGAGA }\end{array}$ & 60 & 467 \\
\hline Exon 7 & $\begin{array}{l}\text { Forward: GAGCTTGCAGTGAGCTGAGA } \\
\text { Reverse: GGGATGTGATGAGAGGTGGA } \\
\text { EX7F_seq CCTGCTTGCCACAGGTCT (to be used for } \\
\text { sequencing instead of Exon } 7 \text { forward primer) }\end{array}$ & 61.5 & 390 \\
\hline Exon 8-9 & $\begin{array}{l}\text { Forward: GACAAGGGTGGTTGGGAGTA } \\
\text { Reverse: GCCCCAATTGCAGGTAAAAC }\end{array}$ & 60 & 500 \\
\hline
\end{tabular}


Table 4 Cytoplasmic and membranous expression of the proteins in angiogenic versus non-angiogenic non-small cell lung cancers (NSCLCs)

\begin{tabular}{|c|c|c|c|c|}
\hline \multirow[t]{2}{*}{ Protein } & \multicolumn{2}{|c|}{ Intensity percentage score (IPS) of protein expression } & \multirow[t]{2}{*}{$P$ value } & \multirow[t]{2}{*}{ Tumour type with higher expression } \\
\hline & Angiogenic tumours & Non-angiogenic tumours & & \\
\hline HIF1 & $6.22 \pm 0.33$ & $6.00 \pm 0.48$ & 0.87 & - \\
\hline HIF2 & $0.00 \pm 0.00$ & $0.00 \pm 0.00$ & Not applicable & - \\
\hline CA9 cytoplasm & $2.38 \pm 0.16$ & $3.50 \pm 0.23$ & $<0.001$ & Non-angiogenic \\
\hline CA9 membrane & $4.00 \pm 0.40$ & $3.30 \pm 0.88$ & 0.62 & - \\
\hline VEGFA & $8.83 \pm 0.35$ & $7.38 \pm 0.78$ & 0.07 & - \\
\hline TYMP & $3.20 \pm 0.54$ & $2.21 \pm 0.32$ & 0.28 & - \\
\hline KDR & $94.56 \pm 1.64$ & $97.80 \pm 1.54$ & $>0.05$ & - \\
\hline KDRp34 & $10.40 \pm 0.30$ & $10.55 \pm 0.62$ & 0.71 & - \\
\hline $\mathrm{FIH}$ & $9.10 \pm 0.32$ & $10.00 \pm 0.47$ & 0.22 & - \\
\hline PHD1 & $3.74 \pm 0.27$ & $4.20 \pm 0.48$ & 0.47 & - \\
\hline PHD2 & $3.50 \pm 0.30$ & $2.46 \pm 0.53$ & 0.02 & Angiogenic \\
\hline PHD3 & $2.70 \pm 0.09$ & $3.38 \pm 0.17$ & $<0.001$ & Non-angiogenic \\
\hline DLL4vessels & $2.34 \pm 0.10$ & $1.53 \pm 0.09$ & $<0.001$ & Angiogenic \\
\hline TSP stroma & $16.98 \pm 2.75$ & $2.00 \pm 1.74$ & $<0.001$ & Angiogenic \\
\hline CXCR4 & $2.47 \pm 0.20$ & $2.34 \pm 0.31$ & 0.92 & \\
\hline EPHB2 & $7.78 \pm 0.27$ & $9.06 \pm 0.33$ & $<0.01$ & Non-angiogenic \\
\hline EPBH3 cytoplasm & $159.3 \pm 8.84$ & $202.20 \pm 9.95$ & $<0.01$ & Non-angiogenic \\
\hline EPBH3 membrane & $23.83 \pm 7.23$ & $86.51 \pm 12.78$ & $<0.001$ & Non-angiogenic \\
\hline EPHB4 & $7.82 \pm 0.26$ & $7.40 \pm 0.38$ & 0.57 & - \\
\hline SOD1 & $4.36 \pm 0.45$ & $9.39 \pm 4.22$ & 0.27 & - \\
\hline $\mathrm{BCL} 2$ & $1.05 \pm 0.25$ & $0.68 \pm 0.35$ & 0.54 & - \\
\hline FOS & $8.05 \pm 0.37$ & $5.31 \pm 0.55$ & $<0.001$ & Angiogenic \\
\hline EGFR & $203.40 \pm 10.79$ & $166.30 \pm 25.74$ & 0.33 & - \\
\hline EGF & $18.90 \pm 3.03$ & $14.44 \pm 4.15$ & 0.72 & - \\
\hline FGF & $9.20 \pm 0.42$ & $9.72 \pm 0.71$ & 0.74 & - \\
\hline BNIP3 & $6.08 \pm 0.36$ & $5.92 \pm 0.82$ & 0.88 & - \\
\hline P53 & $0.28 \pm 0.12$ & $2.28 \pm 0.56$ & $<0.001$ & Non-angiogenic \\
\hline $\mathrm{PI3}$ & $4.62 \pm 0.36$ & $4.92 \pm 0.61$ & 0.51 & - \\
\hline SP1 & $3.70 \pm 0.37$ & $3.38 \pm 0.55$ & 0.86 & - \\
\hline STAT3 & $8.78 \pm 0.39$ & $7.11 \pm 0.79$ & 0.03 & Angiogenic \\
\hline LON & $102.50 \pm 11.20$ & $134.20 \pm 17.10$ & 0.18 & - \\
\hline MEF2D cytoplasm & $50.11 \pm 4.16$ & $65.67 \pm 9.66$ & 0.25 & - \\
\hline MEF2Dmembrane & $60.00 \pm 7.12$ & $67.50 \pm 12.67$ & 0.47 & - \\
\hline JMY & $1.84 \pm 0.18$ & $2.42 \pm 0.31$ & 0.05 & Non-angiogenic \\
\hline TRAP1 & $4.22 \pm 0.28$ & $5.48 \pm 0.31$ & $<0.01$ & Non-angiogenic \\
\hline GST cytoplasm & $236.20 \pm 7.16$ & $249.80 \pm 11.40$ & 0.23 & - \\
\hline GSTmembrane & $28.44 \pm 5.52$ & $69.38 \pm 14.03$ & $<0.01$ & Non-angiogenic \\
\hline NCAM & $0.40 \pm 0.09$ & $3.92 \pm 0.07$ & $<0.001$ & Non-angiogenic \\
\hline $\mathrm{CHGA}$ & $0.08 \pm 0.04$ & $0.27 \pm 0.14$ & $>0.05$ & - \\
\hline SYP & $0.12 \pm 0.05$ & $0.44 \pm 0.15$ & $>0.05$ & - \\
\hline
\end{tabular}

All data are presented as mean \pm standard error

HIF hypoxia-inducible factor; CA9 carbonic anhydrase 9; VEGFA vascular endothelial growth factor A; TYMP thymidine phosphorylase; KDR vascular endothelial growth factor receptor 2; KDRp34 vascular endothelial growth factor p34; FIH factor-inhibiting HIF; PHD prolyl hydroxylase dehydrogenase; DLL4 Delta-like 4; TSP1 thymidine phosphorylase $1 ; C X C R 4$ chemokine (C-X-C motif) receptor $4 ; E P H$ ephrin; SOD1 superoxide dismutase $1 ; B C L 2$ B cell lymphoma $2 ; F O S$ FBJ murine osteosarcoma viral oncogene homolog; EGF epidermal growth factor; EGFR epidermal growth factor receptor; BNIP3 BCL2/adenovirus E1B $19 \mathrm{kDa}$ interacting protein 3; PI3 peptidase inhibitor 3; SP1 Sp1 transcription factor; STAT3 signal transducer and activator of transcription 3; LON Lon protease; MEF2D myocyte enhancer factor 2D; JMY junctionmediating and regulatory protein, p53 cofactor; TRAP1 TNF receptor-associated protein 1; GST glutathione S-transferase; NCAM neural cell adhesion molecule; CHGA chromogranin A; SYP synaptophysin

- Indicates equal expression 
Table 5 Nuclear expression of the proteins in angiogenic versus non-angiogenic NSCLCs

\begin{tabular}{|c|c|c|c|c|}
\hline \multirow[t]{2}{*}{ Protein } & \multicolumn{2}{|c|}{ Intensity percentage score (IPS) of protein expression } & \multirow[t]{2}{*}{$P$ value } & \multirow[t]{2}{*}{ Tumour type with higher expression } \\
\hline & Angiogenic tumours & Non-angiogenic tumours & & \\
\hline HIF1 & $5.49 \pm 0.31$ & $3.79 \pm 0.39$ & 0.01 & Angiogenic \\
\hline VEGFA & $6.09 \pm 0.52$ & $5.62 \pm 0.32$ & 0.74 & - \\
\hline TYMP & $1.94 \pm 0.22$ & $1.16 \pm 0.21$ & 0.42 & - \\
\hline KDR & $231.20 \pm 8.55$ & $229.80 \pm 9.88$ & $>0.05$ & - \\
\hline KDRp34 & $11.69 \pm 0.16$ & $11.45 \pm 0.39$ & 0.77 & - \\
\hline $\mathrm{FIH}$ & $7.50 \pm 0.35$ & $8.15 \pm 0.72$ & 0.50 & - \\
\hline PHD1 & $3.10 \pm 0.34$ & $2.50 \pm 0.35$ & 0.99 & - \\
\hline PHD2 & $2.56 \pm 0.27$ & $1.41 \pm 0.35$ & 0.02 & Angiogenic \\
\hline PHD3 & $2.18 \pm 0.13$ & $0.65 \pm 0.20$ & $<0.001$ & Angiogenic \\
\hline CXCR4 & $5.13 \pm 0.23$ & $3.72 \pm 0.37$ & $<0.01$ & Angiogenic \\
\hline EPHB2 & $3.26 \pm 0.26$ & $4.91 \pm 0.35$ & $<0.001$ & Non-angiogenic \\
\hline $\mathrm{EPBH} 3$ & $99.27 \pm 7.41$ & $147.30 \pm 9.23$ & $<0.001$ & Non-angiogenic \\
\hline EPHB4 & $5.56 \pm 0.30$ & $5.73 \pm 0.34$ & 0.81 & - \\
\hline SOD1 & $4.21 \pm 0.45$ & $5.00 \pm 0.91$ & 0.44 & - \\
\hline C-FOS & $7.50 \pm 0.52$ & $5.53 \pm 0.75$ & 0.05 & Angiogenic \\
\hline EGF & $235.20 \pm 5.60$ & $214.8 \pm 8.68$ & 0.01 & Angiogenic \\
\hline FGF & $7.51 \pm 0.38$ & $8.68 \pm 0.76$ & 0.11 & - \\
\hline BNIP3 & $3.96 \pm 0.40$ & $0.00 \pm 0.00$ & $<0.001$ & Angiogenic \\
\hline P53 & $2.66 \pm 0.36$ & $2.32 \pm 0.53$ & 0.91 & - \\
\hline $\mathrm{Pl} 3$ & $0.53 \pm 0.13$ & $0.00 \pm 0.00$ & $<0.02$ & Angiogenic \\
\hline SP1 & $5.31 \pm 0.46$ & $4.04 \pm 0.60$ & 0.24 & - \\
\hline STAT3 & $7.80 \pm 0.39$ & $6.31 \pm 0.81$ & 0.09 & - \\
\hline MEF2D & $251 \pm 4.62$ & $243.10 \pm 8.04$ & 0.38 & - \\
\hline JMY & $2.01 \pm 0.17$ & $2.60 \pm 0.27$ & 0.07 & - \\
\hline TRAP1 & $1.57 \pm 0.18$ & $4.58 \pm 0.32$ & $<0.001$ & Non-angiogenic \\
\hline GST & $243.30 \pm 6.15$ & $244.10 \pm 10.91$ & 0.55 & - \\
\hline $\mathrm{CHGA}$ & $0.00 \pm 0.00$ & $0.34 \pm 0.12$ & $>0.05$ & - \\
\hline
\end{tabular}

All data are presented as mean \pm standard error

HIF hypoxia-inducible factor; CA9 carbonic anhydrase 9; VEGFA vascular endothelial growth factor A; TYMP thymidine phosphorylase; KDR vascular endothelial growth factor receptor 2; KDRp34 vascular endothelial growth factor p34; FIH factor-inhibiting HIF; PHD prolyl hydroxylasedehydrogenase; DLL4 Delta-like 4; TSP1 thymidine phosphorylase $1 ; C X C R 4$ chemokine (C-X-C motif) receptor $4 ; E P H$ ephrin; SOD1 superoxide dismutase $1 ; B C L 2$ B cell lymphoma 2; FOS FBJ murine osteosarcoma viral oncogene homolog; EGF epidermal growth factor; EGFR epidermal growth factor receptor; $B N I P 3 \mathrm{BCL2} /$ adenovirus E1B $19 \mathrm{kDa}$ interacting protein 3; PI3 peptidase inhibitor 3; SP1 Sp1 transcription factor; STAT3 signal transducer and activator of transcription 3; LON Lon protease; MEF2D myocyte enhancer factor 2D; JMY junctionmediating and regulatory protein, p53 cofactor; TRAP1 TNF receptor-associated protein 1; GST glutathione S-transferase; NCAM neural cell adhesion molecule; CHGA chromogranin A; SYP synaptophysin

- Indicates equal expression

of HIF-controlled genes but lower levels of proteins involved in stromal cell-cell signaling and adhesion, integrins, and Delta-Notch- and EGF-related signaling.

Gene ontology analysis confirmed that proteins usually associated with angiogensis were present in both types of tumours, whereas higher levels of proteins associated with Notch, extracellular matrix, and cell adhesion were present in the truly angiogenesis-producing tumours. However, in the tumours that grow in a nonangiogenic fashion, proteins associated with mitochondria, cell biogenesis, carbonic dehydratase, ephrins, and axon guidance-related functions were more commonly detected (Table 8).

\section{Sequencing}

The higher level of $p 53$ expression in the cytoplasm of the non-angiogenic tumours, as compared with the angiogenic tumours, was one of the most striking results (Table 9) and raised the question of whether non-angiogenic cells had wild-type $p 53$ or a different set of mutations from the angiogenic tumours. Our results showed that p53mutations were observed in eight of 25 angiogenic cases (32.0\%) but 
Table 6 Protein biomarker expression in angiogenic and non-angiogenic NSCLCs according to their intracellular localization

\begin{tabular}{|c|c|c|}
\hline Expression status & $\begin{array}{l}\text { Intracellular } \\
\text { expression localization }\end{array}$ & Proteins \\
\hline \multirow[t]{3}{*}{ Equal expression in both tumour types } & Nuclear & $\begin{array}{l}\text { VEGFA, TYMP, KDR, KDRp34, FIH, EPHB4, PHD1, SOD1, FGF, P53, SP1, } \\
\text { STAT3, MEF2D, JMY, GST, CHGA }\end{array}$ \\
\hline & Cytoplasm & $\begin{array}{l}\text { HIF1, HIF2, VEGFA, TYMP, KDR, KDRp34, FIH, PHD1, SOD1, CXCR4, EPHB4, } \\
\text { BCL2, EGF, EGFR, FGF, BNIP3, PI3, SP1, LON, MEF2D, GST, CHGA, SYP }\end{array}$ \\
\hline & Membrane & CA9, MEF2D \\
\hline \multirow{3}{*}{$\begin{array}{l}\text { Higher expression in angiogenic tumours than } \\
\text { in non-angiogenic tumours in at least one } \\
\text { subcellular location }\end{array}$} & Nuclear & HIF1, PHD2, PHD3, CXCR4, BNIP3, PI3, EGF, FOS \\
\hline & Cytoplasm & PHD2, TSP (stroma), DLL4 (endothelium), FOS, STAT3 \\
\hline & Membrane & None \\
\hline \multirow{3}{*}{$\begin{array}{l}\text { Higher expression in non-angiogenic tumours } \\
\text { than in angiogenic tumours in at least one } \\
\text { subcellular location }\end{array}$} & Nuclear & EPHB2, EPHB3,TRAP1 \\
\hline & Cytoplasm & CA9, PHD3, EPHB2, EPHB3, NCAM, P53, TRAP1, JMY \\
\hline & Membrane & EPHB3, GST \\
\hline
\end{tabular}

HIF hypoxia-inducible factor; CA9 carbonic anhydrase 9; VEGFA vascular endothelial growth factor A; TYMP thymidine phosphorylase; KDR vascular endothelial growth factor receptor 2; KDRp34 vascular endothelial growth factor p34; FIH factor-inhibiting HIF; PHD prolyl hydroxylase dehydrogenase; DLL4 Delta-like 4; TSP1 thymidine phosphorylase $1 ; C X C R 4$ chemokine (C-X-C motif) receptor $4 ; E P H$ ephrin; SOD1 superoxide dismutase $1 ; B C L 2$ B cell lymphoma 2; FOS FBJ murine osteosarcoma viral oncogene homolog; EGF epidermal growth factor; EGFR epidermal growth factor receptor; BNIP3 BCL2/adenovirus E1B $19 \mathrm{kDa}$ interacting protein 3; PI3 peptidase inhibitor 3; SP1 Sp1 transcription factor; STAT3 signal transducer and activator of transcription 3; LON Lon protease; MEF2D myocyte enhancer factor 2D; JMY junctionmediating and regulatory protein, p53 cofactor; TRAP1 TNF receptor-associated protein 1; GST glutathione S-transferase; NCAM neural cell adhesion molecule; CHGA chromogranin A; SYP synaptophysin

Table 7 Selection of visualized pathways

\begin{tabular}{|c|c|c|c|c|c|}
\hline \multicolumn{2}{|c|}{$\begin{array}{l}\text { Proteins equally expressed in angiogenic } \\
\text { and non-angiogenictumours }\end{array}$} & \multicolumn{2}{|c|}{ Proteins up-regulated in angiogenic tumours } & \multicolumn{2}{|l|}{$\begin{array}{l}\text { Proteins up-regulated in } \\
\text { non-angiogenic tumours }\end{array}$} \\
\hline Pathway & Databases & Pathway & Databases & Pathway & Databases \\
\hline Focal adhesion & KEGG, WikiPathways & Focal adhesion & KEGG, WikiPathways & - & - \\
\hline VEGF-related & KEGG, Panther, Reactome & - & - & - & - \\
\hline Angiogenesis & Panther, WikiPathways & Angiogenesis & Panther & Angiogenesis & Panther \\
\hline- & - & Itegrin signalling & Panther & - & - \\
\hline Oxidative stress & Panther, WikiPathways & - & - & - & - \\
\hline Cell response to stress & Reactome & - & - & - & - \\
\hline- & - & Delta-Notch & WikiPathways & - & - \\
\hline- & - & Collagen-related pathways & Reactome & - & - \\
\hline- & - & Cell-cell communication & Reactome & - & - \\
\hline \multirow[t]{2}{*}{-} & - & Integrin signaling & Panther & - & - \\
\hline & & EGF-related pathways & WikiPathways, Panther & - & - \\
\hline- & - & - & - & Response to Hypoxia & Reactome \\
\hline Ephrins signaling & Reactome & - & - & Ephrins-related pathways & Reactome \\
\hline- & - & - & - & Regulation of HIF by oxygen & Reactome \\
\hline- & - & - & - & Regulation of genes by HIF & Reactome \\
\hline- & - & - & - & Nitrogen metabolism & KEGG \\
\hline
\end{tabular}

in five of seven non-angiogenic cases (71.4\%) $(P=0.091$ by Fisher's two-tailed exact test) (Table 9).

All of the detected mutations were heterozygous, and almost all of them corresponded to hot spots previously reported in different tumour types (http://genetics.bwh. harvard.edu/ggi/pph2/c2ea64efde6f039a5ca76a2a264ae 4f3cf922360/1121012.html). The locations of the mutations were randomly distributed across the sequenced region. No specific pattern of mutation location seemed to be related to tumour subtype, angiogenic or non-angiogenic (Fig. 1 and Table 9).

\section{Discussion}

As initially suggested by our mRNA profiling work [19] and also confirmed by the immunohistochemical data presented here, we failed to reveal significant differences 
Table 8 Selection of visualized ontologies

\begin{tabular}{|c|c|c|c|c|c|}
\hline \multicolumn{2}{|c|}{$\begin{array}{l}\text { Proteins equally expressed in angiogenic } \\
\text { and non-angiogenic tumours }\end{array}$} & \multicolumn{2}{|c|}{ Proteins up-regulated in angiogenic tumours } & \multicolumn{2}{|c|}{$\begin{array}{l}\text { Proteins up-regulated in non-angiogenic } \\
\text { tumours }\end{array}$} \\
\hline Ontology & $\begin{array}{l}\text { GO-ontology } \\
\text { database }\end{array}$ & Ontology & $\begin{array}{l}\text { GO-ontology } \\
\text { database }\end{array}$ & Ontology & $\begin{array}{l}\text { GO-ontology } \\
\text { database }\end{array}$ \\
\hline $\begin{array}{l}\text { Cell migration/sprout- } \\
\text { ing angiogenesis } \\
\text { GO.0002042 }\end{array}$ & Biological process & $\begin{array}{l}\text { Immune response- } \\
\text { activating signals } \\
\text { G0.0002757 }\end{array}$ & Biological process & $\begin{array}{l}\text { Regulation of synapses } \\
\text { GO.0051965 }\end{array}$ & Biological process \\
\hline $\begin{array}{l}\text { Endothelial cell } \\
\text { migration } \\
\text { G0.0043534 }\end{array}$ & Biological process & $\begin{array}{l}\text { Immune response-reg- } \\
\text { ulating cell signaling } \\
\text { G0.0002768 }\end{array}$ & Biological process & $\begin{array}{l}\text { Axon guidance and } \\
\text { neuronal regulation } \\
\text { GO.0031290 } \\
\text { GO.0007413 } \\
\text { GO.0021952 } \\
\text { GO.0021955 } \\
\text { GO.0008038 }\end{array}$ & Biological process \\
\hline $\begin{array}{l}\text { VEGF signaling path- } \\
\text { ways } \\
\text { GO.0038084 }\end{array}$ & Biological process & $\begin{array}{l}\text { Mesodermal cell dif- } \\
\text { ferentiation } \\
\text { G0.0048333 }\end{array}$ & Biological process & - & - \\
\hline- & - & $\begin{array}{l}\text { Activation of immune } \\
\text { response } \\
\text { G0.0002253 }\end{array}$ & Biological process & - & - \\
\hline- & - & - & - & $\begin{array}{l}\text { Positive regulation cell } \\
\text { biogenesis } \\
\text { G0.0044089 }\end{array}$ & Biological process \\
\hline- & - & $\begin{array}{l}\text { Basement membrane } \\
\text { GO.0005604 }\end{array}$ & Cell component & - & - \\
\hline- & - & $\begin{array}{l}\text { Extracellular matrix part } \\
\text { GO.0044420 }\end{array}$ & Cell component & $\begin{array}{l}\text { Mitochondrial inter- } \\
\text { membrane space } \\
\text { and matrix } \\
\text { GO.0005758 } \\
\text { G0.0005759 }\end{array}$ & Cell component \\
\hline- & - & $\begin{array}{l}\text { Complex involve in cell } \\
\text { adhesion } \\
\text { G0.0098636 }\end{array}$ & Cell component & - & - \\
\hline $\begin{array}{l}\text { PDGF receptor-binding } \\
\text { G0.0005161 }\end{array}$ & Molecular function & $\begin{array}{l}\text { Fibronectin- and } \\
\text { extracellular matrix- } \\
\text { binding } \\
\text { G0.0001968 } \\
\text { G0.0050840 }\end{array}$ & Molecular function & $\begin{array}{l}\text { Ephrin receptor activity } \\
\text { GO.0005003 }\end{array}$ & Molecular function \\
\hline $\begin{array}{l}\text { Extracellular matrix- } \\
\text { binding } \\
\text { GO.0050840 }\end{array}$ & Molecular function & $\begin{array}{l}\text { Notch-binding } \\
\text { GO.0005112 }\end{array}$ & Molecular function & $\begin{array}{l}\text { Carbonate dehydratase } \\
\text { activity } \\
\text { G0.0004089 }\end{array}$ & Molecular function \\
\hline- & - & - & - & $\begin{array}{l}\text { Axon guidance } \\
\text { GO.0008046 }\end{array}$ & Molecular function \\
\hline
\end{tabular}

between angiogenic and non-angiogenic NSCLCs as far as the expression of proteins associated with the classic hypoxia/angiogenesis pathway is concerned. Because neovascularisation is found in some tumours but not in others, we suggest that activation of the classical angiogenic pathways is necessary, but not sufficient, to induce the sprouting of new vessels in cancer.

The higher levels of expression of proteins associated with extracellular matrix, cell adhesion, and inflammation were in agreement with the observed presence of tumour-associated stroma and chronic inflammation in many angiogenic tumours $[18,22]$. mRNA profiling of these tumours has equally shown that stromal remodeling, cell adhesion, and inflammation are enhanced in angiogenesis [19]. Both mRNA and immunohistochemical data suggest a crucial role in angiogenic cancers for FBJ murine osteosarcoma viral oncogene homolog (FOS), a protein involved in cell proliferation, remodeling, and inflammation. The question remains of whether tissue remodeling is a consequence or a cause of the triggering of angiogenesis. Because non-angiogenic tumours usually preserve the pre-existing architecture, we hypothesize that the triggering of tissue destruction could be a secondary step necessary for the activation of angiogenesis. 
Table 9 Summary of $p 53$ mutations detected in non-angiogenic and angiogenic NSCLC cases

\begin{tabular}{llllll}
\hline Sample ID & Sample type & Mutation location & Mutation type & Domain & Predicted effect on protein activity \\
\hline 104 & Non-angiogenic & c.761T>TA; p.I254S & Missense & HCD IV & Damaging \\
105 & Non-angiogenic & c.734G>GA; p.G245D & Missense & HCD IV & Damaging \\
121 & Non-angiogenic & c.488A>AG; p.Y163C & Missense & DNA binding & Damaging \\
152 & Non-angiogenic & c.634T>TG; p.F212V & Missense & DNA binding & Benign \\
249 & Non-angiogenic & c.314G>GA; p.G105D & Missense & DNA binding & Damaging \\
133 & Angiogenic & c.511G>GT; p.E171X & Nonsense (truncated protein) & HCD III & Truncating \\
138 & Angiogenic & c.824G>GA; p.C275Y & Missense & HCD IV & Damaging \\
139 & Angiogenic & c.het_del216C; p.V73 Wfs48X & Frameshift (truncated protein) & Prolinerich & Truncating \\
141 & Angiogenic & c.407A>AC; p.Q136P & Missense & HCD II & Damaging \\
98 & Angiogenic & c.524G>GA; p.R175H & Missense & HCD III & Potentially damaging \\
147 & Angiogenic & c.524G>GA; p.R175H & Missense & DNA binding & Damaging \\
274 & Angiogenic & c.471_472TC>GA; p.V157G & Missense &
\end{tabular}

Affected domains are listed. The software used to predict the functional effect of the detected sequence changes was PolyPhen_2 (http://genetics.bwh.harvard.edu/ ggi/pph2/c2ea64efde6f039a5ca76a2a264ae4f3cf922360/1121012.html)

$H C D$ highly conserved domain

Fig. 1 Localization of the mutations detected on the $p 53$ gene.
The sequenced region is indicated with an orange line in the figure
above. Mutation locations are indicated with arrowheads (purple
mutations found in angiogenic samples; gray: mutations found in
into five domains, each corresponding to specific functions: yellow is
the highly conserved domain I (HCD I)/transactivation domain; red is
the second transactivation domain, which is proline-rich; blue is the
DNA-binding domain essential for $p 53$-DNA interactions that also
contains HCD II- $V$ and is the target of 90\% of the $p 53$ mutations found
in human cancers; green is the nuclear export signal (NES) localized in
the oligomerization domain of $p 53$

Nonetheless, this process would be far from a general rule because we have observed, in a subset of NSCLC, that angiogenesis can occur in the absence of tissue destruction [23]. Clearly, other mechanisms must exist.

If classical angiogenesis pathways are similarly active in both angiogenic and non-angiogenic tumours, how does their biology differ? Our previous transcriptional profiling work demonstrated higher levels of mRNA coding for molecules associated with oxidative phosphorylation and mitochondrial biogenesis in non-angiogenic tumours [19]. In the present study, we did not examine components of the oxidative phosphorylation pathway, although we did investigate the expression of some proteins involved in mitochondrial functions. Our results showed that proteins related to mitochondria and to cell biogenesis-promoting processes were more highly expressed in non-angiogenic tumours than in angiogenic tumours. This feature was consistent with higher mitochondrial regulatory activity and a possible metabolic switch in non-angiogenic tumours. Again, whether this switch is a cause or an effect of HIF activation remains unclear.

Interestingly, our data suggested increased involvement of response to hypoxia and to HIF regulation by oxygen in non-angiogenic tumours. We hence speculate that, in these tumours, although the HIF pathway failed to induce new vessel formation, it could well be involved in metabolic reprogramming.

Finally, we noted markedly higher levels of cytoplasmic P53 expression in non-angiogenic tumours than in angiogenic tumours via immunohistochemistry. In a pilot study, we sequenced $p 53$ in a limited number of angiogenic and non-angiogenic NSCLCs. Non-angiogenic tumours had a higher incidence of mutations, which were all missense mutations, whereas angiogenic tumours had an amalgam of frame shifts and missense and nonsense mutations. Because $p 53$ also affects mitochondrial respiration [24], it will be necessary to investigate further how the observed $p 53$ mutations could functionally affect its ability to regulate respiration and/or angiogenesis.

\section{Conclusions}

On the basis of our observations collected so far, from the mRNA profiling, immunohistochemical and histopathologic data, we conclude that all tumours, angiogenic and non-angiogenic, experience hypoxia/HIF and VEGF pathway activation. However, angiogenesis does not always ensue. Based on these findings, we suggest that in non-angiogenic tumours, HIF pathway activation could be the driving force toward metabolic reprogramming. 


\section{Additional file}

Additional file 1. Visualization of ontologies and pathways likely to be associated with the patterns of protein expression as detected by immunohistochemistry on angiogenic versus non-angiogenic non-small cell lung carcinomas (NSCLCS). The results represent the combined score, which is computed by the Enrich application by taking the log of the $P$ value from the Fisher's exact test and multiplying it by the $z$ score of the deviation from the expected rank. The length of the bar represents the significance of that specific geneset or term. In addition, the brighter the color is, the more significant that term is. The following are the original results classified according to the databases used (KEGG 2015, Panther, Reactome 2015, WikiPathways 2015, GO-Biological Process, GO-Cell Component, and GO-Molecular Function).

\section{Authors' contributions}

OA designed the study with FP, contributed most of the writing of the manuscript and the laboratory work; RL helped with immunohistochemical staining and data analysis; MFM provided training and technical support for sequencing; $\mathrm{H}, \mathrm{CS}, \mathrm{KCG}$, and $\mathrm{ALH}$ helped with reviewing the literature and the writing of the manuscript; FP designed the study and contributed to most of the writing along with OA. All authors read and approved the final manuscript.

\section{Author details}

${ }^{1}$ Radcliffe Department of Medicine, Nuffield Division of Laboratory Science, John Radcliffe Hospital, University of Oxford, Oxford OX3 9DU, UK. ${ }^{2}$ Radcliffe Department of Medicine, Leukaemia and Lymphoma Research Molecular Haematology Unit, Nuffield Division of Laboratory Science, John Radcliffe Hospital, Oxford OX3 9DU, UK. ${ }^{3}$ Biodonostia Research Institute, Oncology Area, San Sebastian, Spain. ${ }^{4}$ Department of Medical Oncology, Molecular Oncology Laboratories, Weatherall Institute of Molecular Medicine, John Radcliffe Hospital, Oxford OX3 9DU, UK.

\section{Acknowledgements}

OA was supported by a scholarship from the Pathological Society of Great Britain and Ireland. MFM was supported by Leukaemia and Lymphoma Research program (UK) and Associacion Espanola Contra el Cancer (AECC, Spain).

\section{Competing interests}

The authors declare that they have no competing interests.

\section{Ethical approval}

C02.216 The pathobiology of neoplasia in human tissues.

Received: 21 October 2015 Accepted: 20 December 2015

Published online: 12 February 2016

\section{References}

1. Warburg O. The metabolism of tumours in the body. J Gen Physiol 1927:8:519-30.

2. Dupuy F, Tabaries S, Andrzejewski S, Dong Z, Blagih J, Annis MG, et al. PDK1-dependent metabolic reprogramming dictates metastatic potential in breast cancer. Cell Metab. 2015;22:1-13.

3. Warburg O. On the origin of cancer cells. Science. 1956;123(3191):309-14.

4. Pollard PJ, Wortham NC, Tomlinson IP. The TCA cycle and tumorigenesis: the examples of fumarate hydratase and succinate dehydrogenase. Ann Med. 2003;35(8):632-9.

5. Gatenby RA, Gillies RJ. Why do cancers have high aerobic glycolysis? Nat Rev Cancer. 2004;4(11):891-9. doi:10.1038/nrc1478.

6. Frezza C, Gottlieb E. Mitochondria in cancer: not just innocent bystanders. Semin Cancer Biol. 2009;19(1):4-11. doi:10.1016/j.semcancer.2008.11.008.

7. Vander Heiden MG, Cantley LC, Thompson CB. Understanding the Warburg effect: the metabolic requirements of cell proliferation. Science. 2009;324(5930):1029-33. doi:10.1126/science.1160809.
8. Hanahan D, Weinberg RA. Hallmarks of cancer: the next generation. Cell. 2011;144(5):646-74. doi:10.1016/j.cell.2011.02.013.

9. Harris AL. Hypoxia-a key regulatory factor in tumour growth. Nat Rev Cancer. 2002;2(1):38-47. doi:10.1038/nrc704.

10. Denko NC. Hypoxia, HIF1 and glucose metabolism in the solid tumour. Nat Rev Cancer. 2008;8(9):705-13. doi:10.1038/nrc2468

11. Kerbel RS. Tumor angiogenesis. N Engl JMed. 2008;358(19):2039-49. doi:10.1056/NEJMra0706596.

12. Jose C, Bellance N, Rossignol R. Choosing between glycolysis and oxidative phosphorylation: a tumor's dilemma? Biochim Biophys Acta. 2011;1807(6):552-61. doi:10.1016/j.bbabio.2010.10.012.

13. Schulze A, Harris AL. How cancer metabolism is tuned for proliferation and vulnerable to disruption. Nature. 2012;491(7424):364-73. doi:10.1038/nature11706.

14. Yuneva MO, Fan TW, Allen TD, Higashi RM, Ferraris DV, Tsukamoto T, et al. The metabolic profile of tumors depends on both the responsible genetic lesion and tissue type. Cell Metab. 2012;15(2):157-70. doi:10.1016/j.cmet.2011.12.015.

15. Obre E, Rossignol R. Emerging concepts in bioenergetics and cancer research: metabolic flexibility, coupling, symbiosis, switch, oxidative tumors, metabolic remodeling, signaling and bioenergetic therapy. Int $J$ Biochem Cell Biol. 2015;59:167-81. doi:10.1016/j.biocel.2014.12.008.

16. Folkman J. Tumor angiogenesis: therapeutic implications. N Engl J Med. 1971;285(21):1182-6. doi:10.1056/NEJM197111182852108.

17. Pezzella F, Di Bacco A, Andreola S, Nicholson AG, Pastorino U, Harris AL. Angiogenesis in primary lung cancer and lung secondaries. Eur J Cancer. 1996;32A(14):2494-500

18. Pezzella F, Pastorino U, Tagliabue E, Andreola S, Sozzi G, Gasparini G, et al. Non-small-cell lung carcinoma tumor growth without morphological evidence of neo-angiogenesis. Am J Pathol. 1997;151(5):1417-23.

19. Hu J, Bianchi F, Ferguson M, Cesario A, Margaritora S, Granone P, et al. Gene expression signature for angiogenic and nonangiogenic nonsmall-cell lung cancer. Oncogene. 2005;24(7):1212-9. doi:10.1038/ sj.onc. 1208242.

20. Bates GJ, Fox SB, Han C, Launchbury R, Leek RD, Harris AL, et al. Expression of the forkhead transcription factor FOXP1 is associated with that of estrogen receptor-beta in primary invasive breast carcinomas. Breast Cancer Res Treat. 2008;111(3):453-9. doi:10.1007/s10549-007-9812-4.

21. Chen EY, Tan CM, Kou Y, Duan Q, Wang Z, Meirelles GV, et al. Enrichr: interactive and collaborative HTML5 gene list enrichment analysis tool. BMC Bioinformatics. 2013;14:128. doi:10.1186/1471-2105-14-128.

22. Ferguson M. Angiogenesis in human lung tumours. Oxford: Oxford University; 2008.

23. Passalidou E, Trivella M, Singh N, Ferguson M, Hu J, Cesario A, et al. Vascular phenotype in angiogenic and non-angiogenic lung non-small cell carcinomas. Br J Cancer. 2002:86(2):244-9. doi:10.1038/sj.bjc.6600015.

24. Matoba S, Kang J, Patino W, Wragg A, Boehm M, Gavrilova O, et al. p53 regulates mitochondrial respiration. Science. 2006;312(5780):1650-3.

Submit your next manuscript to BioMed Central and we will help you at every step:

- We accept pre-submission inquiries

- Our selector tool helps you to find the most relevant journal

- We provide round the clock customer support

- Convenient online submission

- Thorough peer review

- Inclusion in PubMed and all major indexing services

- Maximum visibility for your research

Submit your manuscript at www.biomedcentral.com/submit 